\title{
O LÚDICO COMO INSTRUMENTO FACILITADOR NA HUMANIZAÇÃO DO CUIDADO DE CRIANÇAS HOSPITALIZADAS
}

\author{
Mirna A Ibuquerque Frota ${ }^{1}$, A dryana A guiar Gurgel2 ${ }^{2}$ M irian Calíope Dantas Pinheiro ${ }^{3}$, \\ M ariana Caval cante M artins ${ }^{4}$, Tathiana A Ives N unes Rodrigues Tavares ${ }^{5}$
}

\begin{abstract}
RESUM O: R ealizou-se um estudo descritivo com abordagem quantitativa no qual objetivou-se verificar o lúdico como facilitador na humanização do cuidado da criança hospital izada. Teve como sujeitos 10 crianças na faixa etária de 3 a 6 anos em condições de brincar e conscientes. A coleta dos dados deu-se através de oficinas envolvendo brincadeiras e observação do comportamento das crianças após a participação nas mesmas, sendo utilizado um check list, denominado de "F icha de registro das reações comportamentais". Os dados foram anal isados e interpretados por meio de gráficos. Como resul tado, observou-se que a promoção do brincar na hospitalização infantil pode facilitar, abrindo possibilidades para uma assistência mais criativa e humanizada, reduzindo os ef eitos estressantes. L ogo, verificou-se a importância da utilização do lúdico na prática diária, pelos profissionais da saúde, facilitando assistência à criança hospitalizada.
\end{abstract}

DESCRITORES: Criança; Hospitalização; Jogos e brinquedos.

\section{PLAYING ASA FACILITATOR FOR THE CARE HUMANIZATION OF HOSPITALIZED CHILDREN}

\begin{abstract}
A descriptive study was carried out, with a quantitative approach that aimed to verify playing as a facilitator for the care humanization of hospitalized children. Ten (10) children between 3 and 6 years of age, conscious and in conditions to play, were the subjects. Data collection was performed through workshops involving playing and the observation of the children's behaviors after participating in it; a check list called "Register form of behavioral reactions" was used. The data were analyzed and interpreted by graphs. As a result, it was observed that playing promotion in child hospitalization can facilitate, opening possibilities for more creative and humanized care delivery, reducing the stress effects. Thus, it was verified theimportance of playing use in health professionals' daily practice, facilitating care rendering to the hospitalized child.
\end{abstract}

DESCRIPTORS: Child; Hospitalization; Play and toys.

\section{EL LÚDICO COMO INSTRUMENTO FACILITATOR EN LA HUMANIZACIÓN DEL CUIDADO DE NIÑO HOSPITALIZADO}

RESUMEN: Ha sido realizado un estudio descriptivo con abordaje cuantitativa, com objetivo de evaluar la importancia del lúdico terapéutico en la humanización del cuidado de los niños hospital izados, teniendo como sujetos 10 niños entre 3 e 6 años en condiciones de jugar y conscientes. L os datos fueron recogidos por medio de trabajos envolviendo juegos, utilizándose un check list llamado de "Ficha de registro de las emociones comportamentales". Los datos han sido analizados y interpretados usándose gráficos. Como resultado, ha sido observado que la promoción de jugar en la hospital ización de niños puede ayudar, dando posibilidad a una asistencia más creativa y humanizada, diminuyendo los efechos estresantes. L uego, se ha verificado la importancia de la utilización del lúdico en la práctica diaria, por los profesionales de la salud, facilitando la asistencia al niño hospitalizado.

DESCRIPTORES: Niño; Hospitalización; J uego e juguetes.

\footnotetext{
${ }^{1}$ Enfermeira.D outora. Professora titular do M estrado em Educação em Saúde. Universidade de Fortaleza - UNIFOR.

${ }^{2}$ Terapeuta ocupacional. Enfermeira, M estranda em Saúde Coletiva da UNIFOR.

${ }^{3}$ Enfermeira. Doutora. Professora titular da Graduação em Enfermagem UNIFOR.

${ }^{4}$ Enfermeira. A luna do M estrado em Saúde Coletiva da UNIFOR. B olsista da CA PES.

${ }^{5}$ Enfermeira do Programa Saúde da Família - B oa Viagem-CE.
} 


\section{INTRODUÇÃO}

A hospitalização é uma experiência desagradável para qualquer pessoa e se agrava quando esta é uma criança, que a tudo assiste sem que lhe caiba o poder de iniciativas e decisões. 0 somatório de vivências da criança no confinamento hospitalar é denominado por muitos estudiosos como "hospitalismo" (1,2).

U ma das formas capazes de esclarecer para a criança essa mudança vivenciada atualmente é o uso do lúdico, que pode al iviar temores eansiedades, permitindo que revele 0 que sente e pensa, por intermédio de brincadeiras.

0 brincar emerge como uma tentativa de transformar 0 ambiente hospitalar, em que proporciona condições para minimizar os danos psicológicos advindos do "hospitalismo", facilitando o acesso à atividade simbólica ea elaboração psíquica de vivências do cotidiano. M ediante os jogos simbólicos, a realidade externa pode ser assimilada à realidade interna, neste caso específico, auxiliando a criança hospitalizada a elaborar melhor esse momento(3).

A humanização interliga o cuidar a diversos fatores, sendo primordial nesse período, e valoriza sentimento, cultura e realidade, possibilitando um envolvimento maior entre o profissional e a criança, em todas as dimensões ${ }^{(4)}$. A atividade lúdica, promovefatores significativos para o desenvolvimento psicomotor, cognitivo, social e afetivo da criança, proporcionando um tratamento humanizado.

Quando as crianças brincam, expressam uma experiência própria e pessoal através do desenvolvimento de uma atividade espontânea, agradável, sem objetivos definidos. Por intermédio do brinquedo, a criança passa a interagir com o meio enesse sentido, desenvolverá sua função social. Independente de como ocorrerá essa socialização, seja na confecção de um brinquedo, compartilhando o material ou até mesmo brigando por sua posse, proporcionando melhor desenvolvimento afetivo(5).

0 brinquedo promove o desenvolvimento global da criança, envolvendo atividades como, a dramatização de papéis, possibilitando o diagnóstico do conflito que a criança está vivendo, tendo, portanto função curativa, funcionando como uma "válvula de escape" e conduz à diminuição da ansiedade pela catarse emocional, constituindo a base da técnica da psicoterapia infantil e também do brinquedo terapêutico, que utiliza princípios lúdicos, proporcionando ao observador melhor compreensão das necessidades e sentimentos da criança, podendo inferir significados que a criança é incapaz de verbalizar(6,7).

A creditando queo lúdico contribui para minimizar traumas da doença e hospitalização, permitindo 0 desenvolvimento e crescimento saudável, como também fornecendo subsídios para profissionais da saúde no atendimento infantil. N este estudo o objetivo foi verificar o lúdico como facilitador na humanização do cuidado da criança hospitalilzada.

\section{DESCRIÇÃO DO MÉTODO}

Trata-se de um estudo descritivo, com abordagem quantitativa, desenvolvido em uma unidade pediátrica de uma instituição hospital ar, com finalidade filantrópica, de referência em Oncologia. A área destinada à coleta dos dados compreendeu o próprio leito da criança e 0 ambulatório, durante os meses de abril e maio de 2004.

A população do estudo foi as crianças da unidade pediátrica oncológica, como amostra selecionou-se 10 crianças na faixa etária de 3 a 6 anos (pré-escolar), de ambos os sexos, obedecendo os critérios de inclusão: estar em condições de brincar, consciente e interagindo com o meio ambiente. Assim, crianças portadoras de agravos no quadro clínico, afetando o desenvolvimento cognitivo, foram excluídas da amostra.

Realizou-se a seleção das crianças, com 0 consentimento das mães ou responsáveis, bem como da própria criança. Conforme a Resolução 196/96, da Comissão Nacional de Ética em Pesquisa - CONEP(8), foi esclarecido o objetivo da pesquisa e solicitado a autorização de sua participação, que deveria ser ratificada pela assinatura do Termo de Consentimento Livre e Esclarecido, ressal tando que a criança poderia desistir, a qual quer momento, da oficina terapêutica, sem, com isso, acarretar quaisquer danos.

Para a coleta de dados, desenvolveu-se um instrumento, denominado " $F$ icha de registro das reações comportamentais" (A nexo), contendo duas partes: a primeira com dados de identificação e a segunda contendo dados referentes a comportamentos previsíveis da criança, como, agressão, expressão verbal, expressão de emoção e movimentação do corpo.

Os dados foram coletados em dois momentos, sendo que os relativos à identificação ocorreram antes das oficinas, juntamente com a observação do comportamento das crianças, que teve duração de aproximadamente trinta minutos. A segunda parte da ficha, que contém dados referentes ao comportamento previsível da criança, foi preenchida após as oficinas, 
que tiveram o tempo de duração de uma hora, durante o tempo em que as crianças permaneceram na unidade de internação.

Durante as oficinas com 0 uso do brinquedo, 0 pesquisador permaneceu próximo, sem interferir na brincadeira; participou quando foi solicitado, auxiliando no manuseio dos brinquedos, respondendo aos questionamentos e atendendo-as na satisfação de al guma necessidade fisiológica.

Buscou-se avaliar as respostas das crianças partici pantes da oficina durante uso da atividade lúdica, utilizando-se dos seguintes brinquedos: bonecos de pano, material hospitalar de brinquedo, como termômetro, seringa, estetoscópio, espátula, cuba-rim, tesoura, utensílios domésticos, como pratinhos, panelinhas, mamadeira, colher, copo, livros de história, figuras representativas da família e da equipe hospitalar, papel e lápis de cor, realizando avaliação, antes e depois de cada atividade.

A pós a coleta dos dados, deu-se o início da análise na qual os dados foram listados, agrupados e categorizados, com base na descrição do universo dos comportamentos e respostas comportamentais apresentados pelas próprias crianças, tendo sido feita a tabulação e em seguida, procedeu-se a organização.

\section{RESULTADOS}

Observando comportamento de crianças internadas, foi possível identificar o desconforto e a angústia de estarem em um lugar desconhecido, cercado de pessoas estranhas, conversas diferentes, procedimentos dolorosos, dentre outros, desenvolvendo na criança uma inquietação e ansiedade, tornando-a insegura.

Diante do contexto, o brinquedo por meio da ludoterapia pode proporcionar à criança hospitalizada a oportunidade de desligar-se da realidade vivenciada, minimizando a tensão, proporcionando subsídios para assimilação de novos conhecimentos, compreendendo o que se passa no hospital e podendo esclarecer possíveis conceitos errôneos ${ }^{(9)}$.

A nal isando as Figuras 1 e 2, percebeu-se que à faixa etária das crianças, houve predominância de 6 anos (40\%), vindo em seguida as de 3 anos (30\%), 5 anos (20\%) e 4 anos (10\%) (Figura 1). Com relação à distribuição conforme o sexo, a maioria das crianças, $60 \%$, era do sexo masculino (Figura 2).

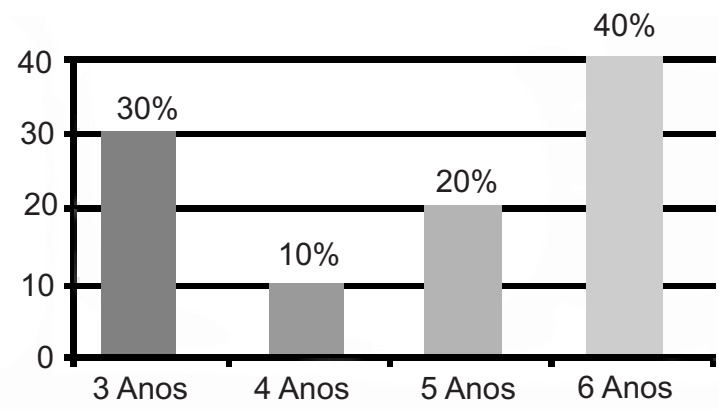

Figura 1- Distribuição das crianças, segundo a idade. Fortal eza, 2004

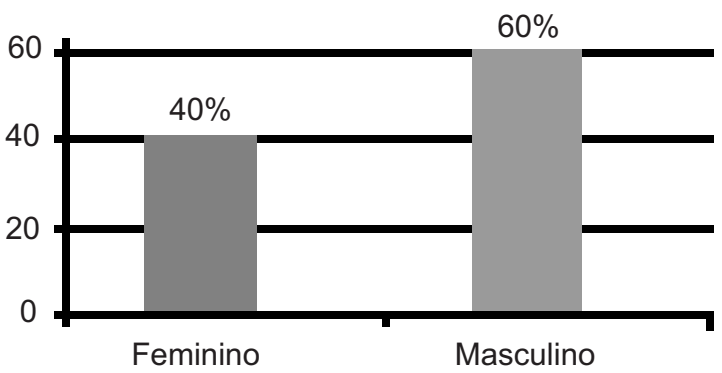

Figura 2 - Distribuição das crianças, segundo 0 sexo. Fortaleza, 2004

Constata-se que houve uma prevalência da faixa etária de 6 anos e no sexo masculino, considerando-se que estudos evidenciam que a incidência de câncer em crianças ocorre mais freqüentemente na faixa etária de 0 a 14 anos, apresentado um pico entre as idades de 3 a 5 anos, sendo mais recorrente em meninos do que em meninas ${ }^{(5,10)}$.

No que concerne às experiências anteriores de hospitalização, houve uma predominância na freqüência de 2 a 3 vezes, total izando $50 \%$ das crianças investigadas, tendo em seguida $30 \%$ com 4 ou mais e $20 \%$ com 1 vez de acordo com a Figura 3.

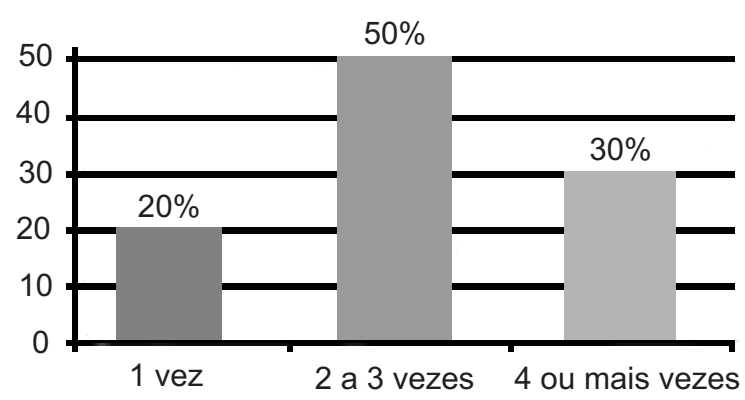

Figura 3 - Distribuição das crianças segundo experiências de hospitalização anteriores. Fortaleza, 2004 
Pode-se constatar que $50 \%$ das crianças apresentaram uma freqüência de 2 a 3 vezes referentes a internações, freqüência essa significativa para uma criança que será submetida a vários procedimentos já conhecidos por el as como mais difíceis de aceitar. Vale ressal tar que em criança vítima do câncer são muito comuns, diversas internações, desde o diagnóstico até o término do tratamento, pois são submetidas a várias seções de quimioterapia, ficando debilitadas e susceptíveis as infecções e outras complicações, resultando em várias hospitalizações ${ }^{(5)}$.

$\mathrm{Na}$ análise do comportamento relacionado a expressões verbais, o qual foi avaliado mediante as falas, os gestos e as ações das crianças, observou-se (Figura 4) que o comportamento identificado como "nega" antes da oficina foi expresso apenas por uma criança (1\%) dado este modificado após novo estimulo, passando para $100 \%$, pois, comportamentos como negar podem indicar que a criança se encontra em estado de tensão.

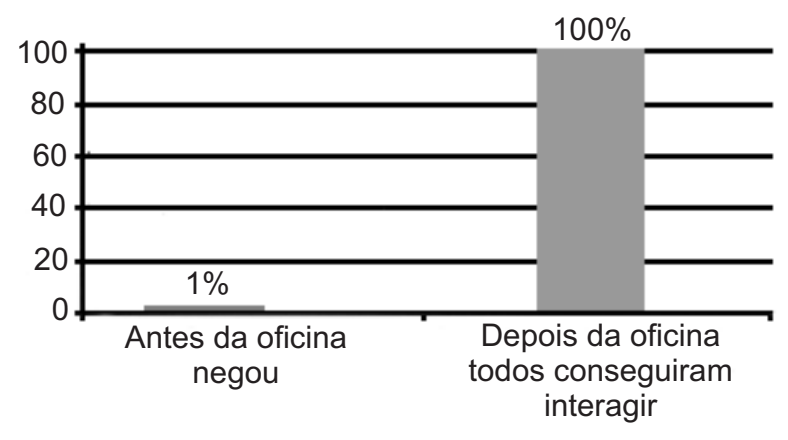

Figura 4 - Crianças, segundo os comportamentos da categoria expressão verbal. Fortal eza, 2004

E m relação à abordagem da expressão verbal, pôde-se constatar um quantitativo menor, pois o lúdico pode proporcionar um momento de descontração, como também uma fuga da realidade vivenciada, pois o brincar, associado ao bem-estar da saúde, expressa os medos e tensões, favorecendo a criança compreender e permitindo a realização de atividades com o brinquedo ${ }^{(11)}$.

A ssim, os profissionais da área da saúde, em especial a enfermagem, devem cuidar da criança de forma holística ampliando seus ol hares para a família, para o cuidador, com a finalidade de amenizar as dúvidas e aflições diante da criança hospitalizada, fortalecendo um vínculo através do diálogo ${ }^{(12)}$.

Evidenciando a expressão da emoção (Figura 5) dentre as crianças investigadas, quatro crianças (40\%) variando na faixa etária de 3 a 4 anos, manifestaram comportamentos de "chora baixinho" antes das oficinas, não se observando após a participação nas oficinas, no restante do tempo em que permaneceu internada. Em seguida, pôde-se perceber que $60 \%$ das crianças, antes das oficinas, apresentaram o comportamento de "sorrir" e depois esse número passou a $80 \%$.

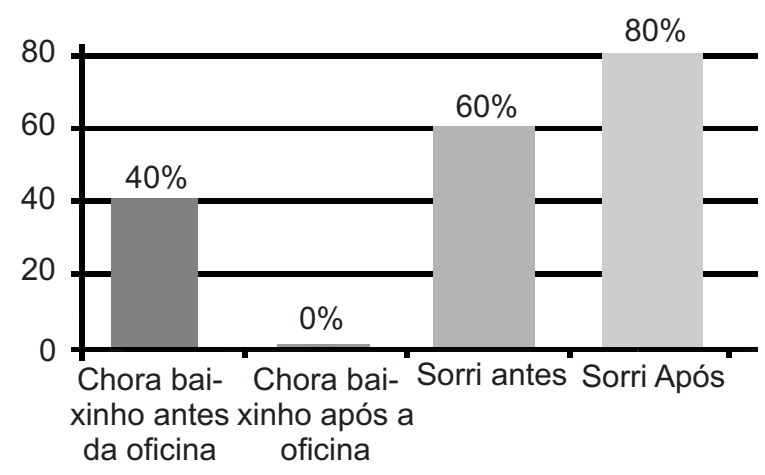

Figura 5 - Crianças, segundo os comportamentos da categoria expressão de emoção. Fortaleza, 2004

Constata-se que crianças na faixa etária de 3 a 4 anos apresentaram dificuldades para expressar seus sentimentos, mesmo fazendo uso do brinquedo, pois a imaturidade para enfrentar situações adversas inibeas. Sabe-se, portanto, que mudança de comportamento não acontece imediatamente, sendo este um processo gradativo, no qual os profissionais devem estar preparados para uma variedade de atitudes inesperadas.

Referindo-se ao movimento do corpo, Figura 6, os comportamentos de "movimentar-se" e "ficar quieta" mostraram dados significantes, podendose perceber que, antes das oficinas, $60 \%$ das crianças movimentavam-se, sem voltar a atenção para o que estava sendo desenvolvido e $40 \%$ ficaram quietas. A pós as oficinas, houve uma mudança nesse quantitativo, havendo evolução, sendo que $30 \%$ das crianças se movimentavam e $70 \%$ ficavam quietas, tendo a atenção voltada para as atividades desenvolvidas durante a oficina. No presente estudo, esses resultados demonstram a efetividade de oficinas terapêuticas no âmbito hospitalar, porque possibilitam maior capacidade de interação e concentração, facilitando a quietude após a oficina.

Evidenciando-se, a Figura 6, notou-se a importância da presença da atividade lúdica durante 
o período de internação, pois a criança compreende que pode fazer aos outros o que lhe foi feito, brincando com instrumentos utilizados pela equipe de enfermagem como seringa, termômetros, tesoura e etc, através da dramatização de situações vivenciadas durante a hospitalização, podendo amenizar a tensão sofrida.

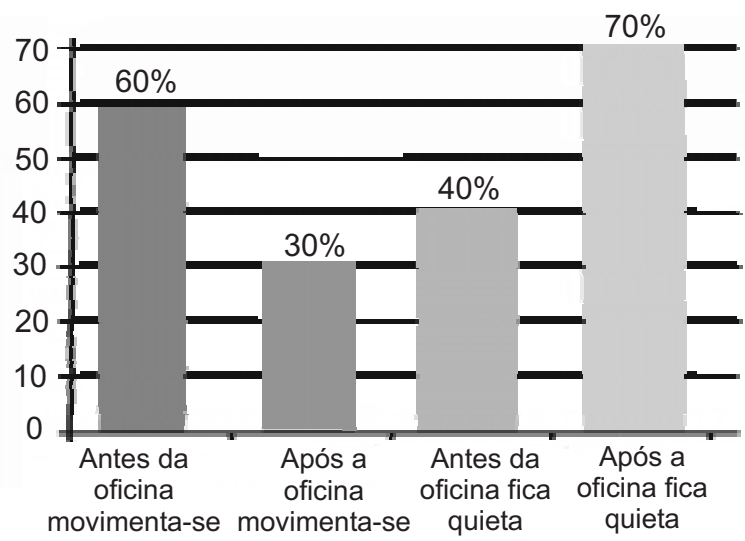

Figura 6 - Crianças, segundo os comportamentos da categoria movimentação do corpo. Fortal eza, 2004

A criança que sofreu na passividade durante a internação revela na brincadeira uma atividade em relação ao sofrimento, em que os acontecimentos traumáticos e todas as suas implicações podem ser dominados, deixando-as mais calmas, e, assim, podem explorar e descobrir de forma acentuada 0 brinquedo(13,14).

Neste sentido, o uso do lúdico mostrou-se um recurso humanizado à disposição da equipe de saúde, para ajudar a criança a lidar com experiências estressantes, ampliando o campo perceptual e permitindo-Ihe exteriorizar sentimentos e conflitos, possibilitando melhor interação com o brinquedo pela redução da agitação corporal, mantendo-se quieta e tranqüila na unidade, após a participação nas oficinas.

Por outro lado, o profissional da saúde deve envolver-se no contexto do lúdico, conscientizandose da importância de tal estratégia, possibilitando uma vivência diferente, em que poderá estabelecer víncul os prazerosos com a criança hospital izada, facilitando 0 tratamento ${ }^{(15,16)}$.

A hospitalização é uma experiência estressante e traumática para a criança, com reflexo no comportamento durante a permanência no hospital, sendo a ludicidade uma alternativa de autoterapia, proporcionando uma possibilidade na resolução dos conflitos existenciais. Este fato só reafirma que a criança, quando brinca, desloca para o exterior seus medos, angústias e problemas interiores.

Nessa perspectiva, o brincar surge como uma possibilidade de expressão de sentimentos, preferências, receios, mediação entre o mundo familiar e situações novas e de transtorno de comportamento, reconhecendo suas singularidades e especificidades e se sentindo descontraída e feliz, tornando sua permanência no hospital mais fácil e favorecendo 0 seu desenvolvimento e cura ${ }^{(2,3,5,17-19)}$.

\section{CONSIDERAÇÕES FINAIS}

$\mathrm{Na}$ tentativa de alcançar a humanização na assistência à criança no processo de hospital ização, a equipe de profissionais de saúde dispõe de instrumento capaz de proporcionar vivências com novos objetos, sensações, atividades e sentimentos não ameaçadores. Desta forma, o brinquedo proporciona a dinâmica de interações sendo sua utilização a articulação entre 0 tratamento e cura da criança hospitalizada.

A criança enfrenta dificuldades com as experiências dolorosas e desagradáveis, portanto, 0 apoio daquel es que assistem a criança é essencial para a adaptação no âmbito hospitalar e tratamentos aos quais é submetida, pois a atuação diferenciada com a criança contribui no cotidiano da internação.

Com isso, cabe salientar que a tríade profissional - brinquedo - criança interliga propósitos e expectativas, facilitando a interação positiva, sendo o brinquedo predominantemente a ferramenta relevante à intervenção humanizada, promovendo 0 movimento entre - mundo real e imaginário transpondo as barreiras do adoecimento. A ssim, fazse necessário o envolvimento no processo terapêutico, minimizando a angústia da criança e familiares, priorizando 0 universo infantil, maximizando 0 potencial de afetividade, amor e vontade de ser companheiro da criança na experiência vivenciada.

\section{REFERENNCIAS}

1. Winnicot DW. A criança e o seu mundo. 6 a ed. Rio de J aneiro: Zahar;1985.

2. Oliveira SSG, Dias M GBB, Roazzi A . O lúdico e suas implicações nas estratégias de regulação das emoções em crianças hospitalizadas. Psicol Reflex Crit 2003; 16(1):1-13.

3. Lindquist I. A criança no hospital - terapia pelo 
brinquedo. São Paulo: Página A berta; 1993.

4. Rolim K M C, Cardoso M V L M L. 0 discurso e a prática do cuidado ao recém-nascido de risco: refletindo sobre a atenção humanizada. Rev Latino-am Enferm 2006; 14(1):85-92.

5. Whaley LF, Wong DL. Enfermagem pediátrica: elementos essenciais à intervenção efetiva. Rio de Janeiro: Guanabara; 1999.

6. Souza SVDE, Camargo DDE, Bulgacov YLM . Expressão da emoção por meio do desenho de uma criança hospitalizada. Psicol Estud 2003; 8(1):101-9.

7. Vygotsky LS. A formação social da mente. São Paulo: M artins Fontes; 1998.

8. M inistério da Saúde (BR ). Conselho N acional de Saúde. Diretrizes e normas regulamentadoras de pesquisa envolvendo seres humanos. Resolução 196, de 10 de outubro de 1996. B rasília; 1996.

9. $M$ artins $M R$, Ribeiro $C A, B$ orba RIH, Silva CV. Protocolo de preparo da criança pré-escolar para punção venosa, com utilização do brinquedo terapêutico. Rev Latino-am Enferm 2001; 9(2):76-85.

10. M arcondes E. Pediatria básica. 8a ed. São Paulo: Sarvier; 1991.

11. Winnicot DW. 0 ambiente e os processos de maturação - estudos sobre a teoria do desenvolvimento emocional. Porto A legre: A rtes M édicas; 1988.

12. Schaurich $D$, Padoin SM M . Experienciando um marco conceitual para o cuidado em enfermagem ao serfamiliar e/ou cuidador eao ser-criança. Cogitare Enferm 2003; 8(2):34-43.

13. Mitre RM . B rincando para viver: um estudo sobre a relação entre a criança gravemente adoecida e hospitalizada e o brincar [dissertação]. Rio de Janeiro (RJ): Instituto Fernandes Figueira, Fiocruz; 2000.

14. Santa RE. Quando brincar é dizer. Rio de Janeiro: Contracapa; 1999.

15. Mitre RM, Gomes R. A promoção do brincar no contexto da hospital ização infantil como ação de saúde. Rev Cien Saúde Col 2004; 9:147-54.

16. Vieira LJES, A raújo $K L, C$ atrib AM F, Vieira ACVC. 0 lúdico na prevenção de acidentes em crianças de 4 a 6 anos. Rev Bras Promo Saúde 2005; 18:78-4.

17. A ngerami-Camon VA, Chiattoni HBC, Sebastiani RW, Fongaro M LH, Santos CT. E a psicologia entrou no hospital. São Paulo: Pioneira; 2001.

18. Friedmann A. B rincar: crescer e aprender. 0 resgate do jogo infantil. São Paulo: M oderna;1996.
19. B retâs JRS, Santos $F Q$, Castro AS, Genovese RE, $M$ artins $M R, M$ anásia $L A H$, Contim D. Contribuições para o cuidado emocional à criança hospitalizada. A cta Paul Enferm 2002; 15:87-4.

Cogitare Enferm 2007 jan/mar; 12(1):69-75 
FICHA DE REGISTRO DAS REAÇÕES COMPORTAMENTAIS

1. IDENTIFICAÇÃO:

Nome:

Idade: Sexo: $F() M(~)$

2. DADOS REFERENTES À DOENÇA:

- Experiências de hospitalizações anteriores: 1 vez ( ) 2 a 3 vezes ( ) 4 ou mais ( )

- Período da hospitalização vigente

3. REGISTRO DOS COMPORTAMENTOS PREVISÍVEIS DA CRIANÇA HOSPITALIZADA ANTES E APÓS AS OFICINAS TERA PÊUTICAS:

\begin{tabular}{|l|c|c|}
\hline \multicolumn{2}{|c|}{ REAÇÕES DA CRIANÇA } \\
\hline 1. AGRESSÃO & ANTES & APÓS \\
\hline Física & & \\
\hline Verbal & ANTES & APÓS \\
\hline 2. EXPRESSÃO VERBAL & & \\
\hline Grita & & \\
\hline Ameaça & & \\
\hline Discute & & APÓS \\
\hline Culpa & & \\
\hline Exige & ANTES & \\
\hline Nega & & APÓS \\
\hline 3. EXPRESSÃO DE EMOÇÃO & & \\
\hline Chora & & \\
\hline Chora baixinho & & \\
\hline Sorri & & \\
\hline 4. MOVIMENTAÇÃO DO CORPO & & \\
\hline Manipula o corpo & & \\
\hline Fica quieta & & \\
\hline Movimenta-se & & \\
\hline
\end{tabular}

